\title{
Origin of spectral purity and tuning sensitivity in a vortex-based spin transfer nano-oscillator
}

\author{
A. Hamadeh, G. de Loubens, ${ }^{*}$ and O. Klein \\ Service de Physique de l'État Condensé (CNRS URA 2464), CEA Saclay, 91191 Gif-sur-Yvette, France \\ V.V. Naletov \\ Service de Physique de l'État Condensé (CNRS URA 2464), CEA Saclay, 91191 Gif-sur-Yvette, France \\ Unité Mixte de Physique CNRS/Thales and Université Paris Sud 11, 1 av. Fresnel, 91767 Palaiseau, France and \\ Institute of Physics, Kazan Federal University, Kazan 420008, Russian Federation \\ N. Locatelli, R. Lebrun, J. Grollier, and V. Cros \\ Unité Mixte de Physique CNRS/Thales and Université Paris Sud 11, 1 av. Fresnel, 91767 Palaiseau, France
}

(Dated: May 28, 2022)

\begin{abstract}
We investigate the microwave characteristics of a spin transfer nano-oscillator (STNO) based on coupled vortices as a function of the perpendicular magnetic field $H_{\perp}$. While the generation linewidth displays strong variations on $H_{\perp}$ (from $40 \mathrm{kHz}$ to $1 \mathrm{MHz}$ ), the frequency tunability in current remains almost constant $(\simeq 7 \mathrm{MHz} / \mathrm{mA})$. We demonstrate that our vortex-based oscillator is quasi-isochronous independently of $H_{\perp}$, so that the severe nonlinear broadening usually observed in STNOs does not exist. Interestingly, this does not imply a loss of frequency tunability, which is here governed by the current induced Oersted field. Nevertheless this is not sufficient to achieve the highest spectral purity in the full range of $H_{\perp}$ either: we show that the observed linewidth broadenings are due to the excited mode interacting with a lower energy overdamped mode, which occurs at the successive crossings between harmonics of these two modes. These findings open new possibilities for the design of STNOs and the optimization of their performance.
\end{abstract}

A spin-polarized current exerts on a ferromagnetic material a torque that can compensate the damping and lead to auto-oscillation of the magnetization [14]. Owing to their tunability, agility, compactness and integrability, spin transfer nano-oscillators (STNOs) are promising candidates for various high frequency applications such as frequency generation, signal processing and microwave frequency detection. Spectral purity and tuning sensitivity are two key characteristics for such devices. A particularity of STNOs compared to other oscillators is their strong nonlinear properties, which are inherited from the equation of motion of magnetization [5. On one hand, they confer interesting properties to STNOs, as for instance their large frequency tunability 4,6 . On the other hand, they lead to a severe broadening of the generation linewidth [7, which is the main limiting factor to their practical applications. In this Letter, we shall demonstrate that strong nonlinearities are not necessary to achieve large tuning sensitivity, while weak nonlinearities are not sufficient to obtain high spectral purity, which points towards alternative routes to engineer STNOs with improved performance.

So far, some of the best microwave characteristics have been reported for STNOs in which spin transfer torque (STT) excites the gyrotropic mode of a magnetic vortex [8]. It results in microwave emission in the range $100 \mathrm{MHz}-2 \mathrm{GHz}$ characterized by a narrow linewidth (about $1 \mathrm{MHz}$ ) and large output power in the case of TMR devices [9]. Moreover, the oscillation frequency of vortex-based STNOs can be rapidly switched between different values using the bias current, which demonstrates their high agility and tunability in current [10]. Recently, we have reported a record high spectral purity (quality factor $Q>15000$ ) in a spin-valve nano-pillar where STT drives the dynamics of two coupled vortices (one in each ferromagnetic layer) [11. In such a STNO, the generation linewidth at fixed dc current $I_{\mathrm{dc}}$ displays strong variations (down from $40 \mathrm{kHz}$ [Fig 17] up to $1 \mathrm{MHz}$ [Fig 1 $1 \mathrm{p}]$ ) on the applied perpendicular field $H_{\perp}$, as displayed in Fig 2 $\mathrm{k}$. At the same time, its frequency tunability $d F / d I_{\mathrm{dc}} \simeq 7 \mathrm{MHz} / \mathrm{mA}$ remains almost constant (see Fig 2 b), which points out that spectral purity and tuning sensitivity are uncorrelated. In the following, we aim at understanding the physical origin of these peculiar behaviors, as this will give hints to optimize the characteristics of STNOs.

The studied STNO is a circular nanopillar of diameter $2 R=250 \mathrm{~nm}$ patterned from a $\left(\mathrm{Cu} 60 \mid \mathrm{Py}_{\mathrm{B}} 15\right.$ $\left.|\mathrm{Cu} 10| \mathrm{Py}_{\mathrm{A}} 4 \mid \mathrm{Au} 25\right)$ stack, where thicknesses are in nm and $\mathrm{Py}=\mathrm{Ni}_{80} \mathrm{Fe}_{20}$, which can sustain the double vortex configuration [11, 12. A current $I_{\mathrm{dc}}>0$ is injected through the STNO using the bottom $\mathrm{Cu}$ and top $\mathrm{Au}$ electrodes, which corresponds to electrons flowing from the thick $\mathrm{Py}_{\mathrm{B}}$ to the thin $\mathrm{Py}_{\mathrm{A}}$ layer. In both Py layers, the vortex chiralities are parallel to the orthoradial Oersted field produced by $I_{\mathrm{dc}}$. We use a magnetic field $H_{\perp}$ perpendicular to the sample plane in order to control the relative orientation of the vortex core polarities. As shown in Ref.[11], we observe a narrow microwave signal only in the case when they are opposite, $p_{\mathrm{A}} p_{\mathrm{B}}=-1$, 


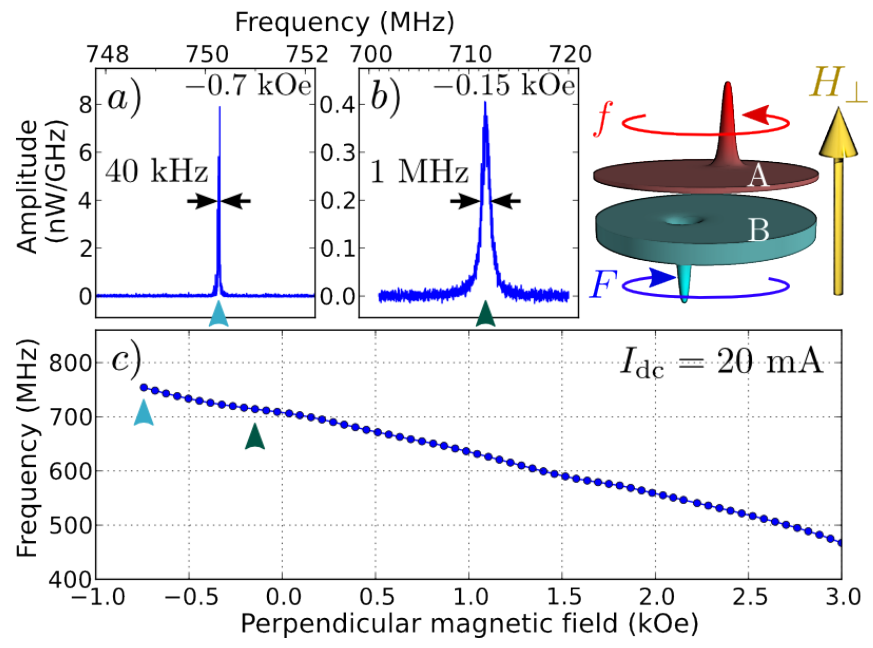

FIG. 1. (Color online) Upper right sketch: STNO based on coupled vortices. (a) Power density spectra measured at $I_{\mathrm{dc}}=$ $20 \mathrm{~mA}$ and a perpendicular magnetic field $H_{\perp}=-0.7 \mathrm{kOe}$ and (b) $H_{\perp}=-0.15 \mathrm{kOe}$. (c) Dependence of the fundamental oscillation frequency on $H_{\perp}$.

and when $I_{\mathrm{dc}}$ exceeds a threshold current $I_{\mathrm{th}} \simeq 8$ to $11 \mathrm{~mA}$ depending on $H_{\perp}$. The spontaneous microwave emission branch at $I_{\mathrm{dc}}=20 \mathrm{~mA}$ for which $p_{\mathrm{A}}=+1$ and $p_{\mathrm{B}}=-1$ is displayed in Fig 1: The oscillation frequency linearly decreases with increasing $H_{\perp}$, as expected for a gyrotropic mode dominated by the thick layer vortex, whose polarity is antiparallel to the applied field [13]. The observed emission frequency, which decreases from $750 \mathrm{MHz}$ to $450 \mathrm{MHz}$ as $H_{\perp}$ increases from $-0.7 \mathrm{kOe}$ to $3 \mathrm{kOe}$, agrees well [1] with the expected gyrotropic frequency for the thick $\mathrm{Py}_{\mathrm{B}}$ layer augmented with the contributions of the Oersted field [14, 15] and of the dipolar coupling to the thin $\mathrm{Py}_{\mathrm{A}}$ layer [16]. The boundaries of the frequency branch shown in Fig 1 1 result from the combined action of $H_{\perp}$ and STT to reverse the vortex cores in the Py layers [17.

We now concentrate on the dependence of the generation linewidth on $H_{\perp}$, which is plotted in Fig 2 a using dark blue symbols $\left(\Delta F_{1}\right)$. It displays minima down to $40 \mathrm{kHz}\left(F_{1} / \Delta F_{1} \simeq 19000\right)$ and maxima up to $1 \mathrm{MHz}\left(F_{1} / \Delta F_{1} \simeq 700\right)$, i.e., a decrease of spectral purity by more than a factor 25 by changing $H_{\perp}$ from $-0.7 \mathrm{kOe}$ [Fig 1 $\mathrm{B}]$ to $-0.15 \mathrm{kOe}$ [Fig 1 $\mathrm{p}]$. We first note that the strong variations of the linewidth observed at $I_{\mathrm{dc}}=20 \mathrm{~mA}$ cannot be attributed to changes of the nearly constant supercriticality $\left(I_{\mathrm{dc}} / I_{\mathrm{th}} \simeq 2\right)$. The generation linewidth of a nonlinear auto-oscillator can be written as [7]:

$$
\Delta F_{\mathrm{g}}=\frac{k_{B} T}{E_{s}} \frac{\Gamma_{+}}{2 \pi}\left(1+\nu^{2}\right),
$$

where $k_{\mathrm{B}}$ is the Boltzmann constant, $T$ the temperature, $E_{s}$ the energy stored in the auto-oscillation, $\Gamma_{+}$the natural energy dissipation rate, and $\nu$ the nonlinear phase-
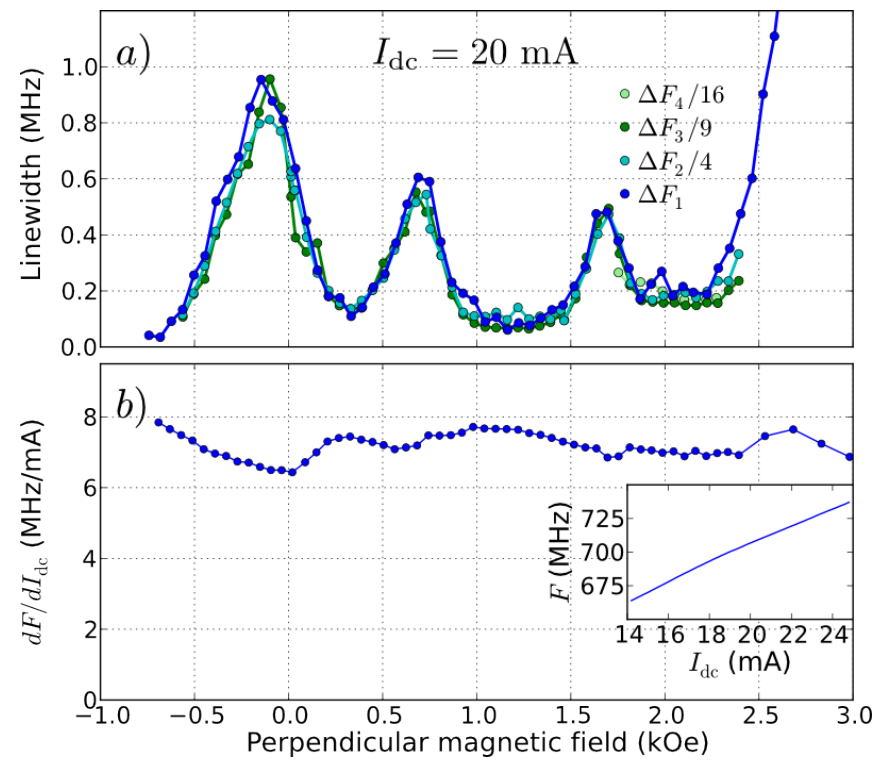

FIG. 2. (Color online) (a) Generation linewidths of harmonics $n=1$ to 4 divided by $n^{2}$ as a function of $H_{\perp}$. (b) Frequency tunability vs. $H_{\perp}$. Inset: dependence of the oscillation frequency on $I_{\mathrm{dc}}$ measured at $H_{\perp}=0 \mathrm{kOe}$.

amplitude coupling parameter. The latter is defined as $\nu=N p / \Gamma_{p}$, the dimensionless ratio between the nonlinear frequency shift $N$ multiplied by the normalized oscillation power $p$ and divided by the damping rate of amplitude fluctuations $\Gamma_{p}[\underline{5}$.

Since the spectral linewidth $\Delta F_{\mathrm{g}}$ depends quadratically on $\nu$, the dependence of this parameter on $H_{\perp}$ should be evaluated. For this, we analyze the linewidth of the harmonics of the auto-oscillation signal. It was shown in Ref. 18] that the linewith $\Delta F_{n}$ of the $n^{\text {th }}$ harmonics is related to the fundamental one $\left(\Delta F_{1}\right)$ by:

$$
\frac{1}{n^{2}}=\Delta F_{1}\left(\frac{1+\nu^{2}}{\Delta F_{n}}-\nu^{2} \frac{1-\exp \left(-2 \Gamma_{p} / \Delta F_{n}\right)}{2 \Gamma_{p}}\right) \text {. }
$$

We have plotted in Fig $2 a$ the evolution of $\Delta F_{n} / n^{2}$ ( $n=2$ to 4$)$ together with the one of $\Delta F_{1}$. It is clear from this graph that independently of $H_{\perp}, \Delta F_{n} \simeq n^{2} \Delta F_{1}$, which means that our STNO is quasi-isochronous and from Eq. 22), that $\nu \simeq 0$ in the full field range. Therefore, one can also exclude that the strong variations of linewidth observed in Fig $2 \mathrm{a}$ are due to some changes of $\nu$ with $H_{\perp}$. Moreover, we can estimate the generation linewidth from Eq.(1) when $\nu=0$. For the vortex gyrotropic mode with angular frequency $\omega, E_{s}=\frac{1}{2} G \omega X^{2}$, where $G=2 \pi L M_{s} / \gamma$ is the gyrovector and $X$ the gyration radius of the core and $\Gamma_{+}=\eta \alpha \omega$ [19]. For the thick $\mathrm{Py}_{\mathrm{B}}$ layer $(L=15 \mathrm{~nm}), M_{s}=764 \mathrm{emu} . \mathrm{cm}^{-3}$, $\gamma=1.87 \cdot 10^{7}$ rad. $\mathrm{s}^{-1}$.Oe $\mathrm{e}^{-1}$ and the damping coefficient $\alpha=0.008$ were determined by mechanical ferromagnetic resonance [20], and $\eta=1.7$ [14]. From micromagnetic simulations [21, the radius of gyration under 
bias conditions close to the experimental ones is found to be $X \simeq 40 \mathrm{~nm}$ (corresponding to $p=(X / R)^{2} \simeq 0.1$ ). Hence, at room temperature, $k_{B} T / E_{s} \simeq 0.003$ and $\Gamma_{+} /(2 \pi)=9.5 \mathrm{MHz}$, which yields $\Delta F_{g}=29 \mathrm{kHz}$, a value close to the narrow linewidth of $40 \mathrm{kHz}$ observed at $H_{\text {ext }}=-0.7 \mathrm{kOe}[\mathrm{Fig}, 1 \mathrm{~A}]$, which thus almost coincides with the intrinsic linewidth of our oscillator. In sum, the absence of nonlinear broadening and the large energy stored in the auto-oscillation in comparison to the thermal energy explain the low minimal values $(<100 \mathrm{kHz})$ of the linewidth in Fig, 2 .

Before seeking further the origin of the variations of linewidth, we first investigate possible physical causes of quasi-isochronicity. Despite the fact that the nonlinearity $\nu$ of our STNO is nearly zero, it exhibits a large frequency tunability, $d F / d I_{\mathrm{dc}} \simeq 7 \mathrm{MHz} / \mathrm{mA}$, see Fig $2 \mathrm{~b}$. This can be explained by the linear contribution of the Oersted field to the oscillation frequency. In fact, the tunability can be decomposed as follows:

$$
\frac{d F}{d I_{\mathrm{dc}}}=\frac{\partial F}{\partial p} \frac{\partial p}{\partial I_{\mathrm{dc}}}+\frac{\partial F}{\partial I_{\mathrm{dc}}}=N \frac{\partial p}{\partial I_{\mathrm{dc}}}+A_{\mathrm{Oe}} I_{\mathrm{dc}} .
$$

In the vortex state, the Oersted field participates to the confinement potential of the vortex core [14, 15], and for our STNO parameters, it is predicted to be about $A_{\mathrm{Oe}} \simeq 12.5 \mathrm{MHz} / \mathrm{mA}$. Therefore, a nonlinear frequency shift $N \neq 0$ is not required to obtain a large tuning sensitivity in Eq. (3). Here, the measured tunability is comparable with the one expected for the Oersted field alone. We attribute the somewhat smaller experimental value to the fact that the auto-oscillating mode does not only involve the thick Py layer, but also the thin one [11, which is not taken into account in the calculation of $A_{\mathrm{Oe}}$.

For the gyrotropic mode, $N$ is the sum of the nonlinearities $N_{\mathrm{ms}}$ and $N_{\mathrm{Oe}}$ of the magnetostatic and the Oersted field confinement potentials, respectively. An analytical model 22] predicts that the exact value of $N_{\mathrm{ms}}$ is positive and depends on the aspect ratio of the magnetic dot in the vortex state, as confirmed by recent micromagnetic simulations [15] and experiments [23]. On the contrary, $N_{\text {Oe }}$ is negative when the vortex chirality is parallel to the Oersted field. Therefore, the two contributions can compensate each other (see Fig.3 of Ref. 15]): using our STNO parameters, $N=N_{\mathrm{ms}}+N_{\mathrm{Oe}}=0$ for $I_{\mathrm{dc}}=23 \mathrm{~mA}$. Experimentally, we find that $\nu \simeq 0$ is robust from $18 \mathrm{~mA}$ up to $25 \mathrm{~mA}$ by analyzing the linewidths of harmonics as in Fig.2a. By using Eq.(2), we can be more quantitative [18: in the full window of field and current, we extract that $\nu<0.5$ and that $\Gamma_{p}$ ranges between 2 and $10 \mathrm{MHz}$. Hence, the intrinsically small $N$ with respect to $\Gamma_{p} / p \simeq 10 \cdot \Gamma_{p}$ in our STNO is probably responsible for $\nu<0.5$ 24. Moreover, as mentioned before, the autooscillating mode in our sample involves both the thick and thin vortex Py layers with opposite core polarities. Several non-conservative STT terms are thus involved in the dynamics [25, which could be another cause of
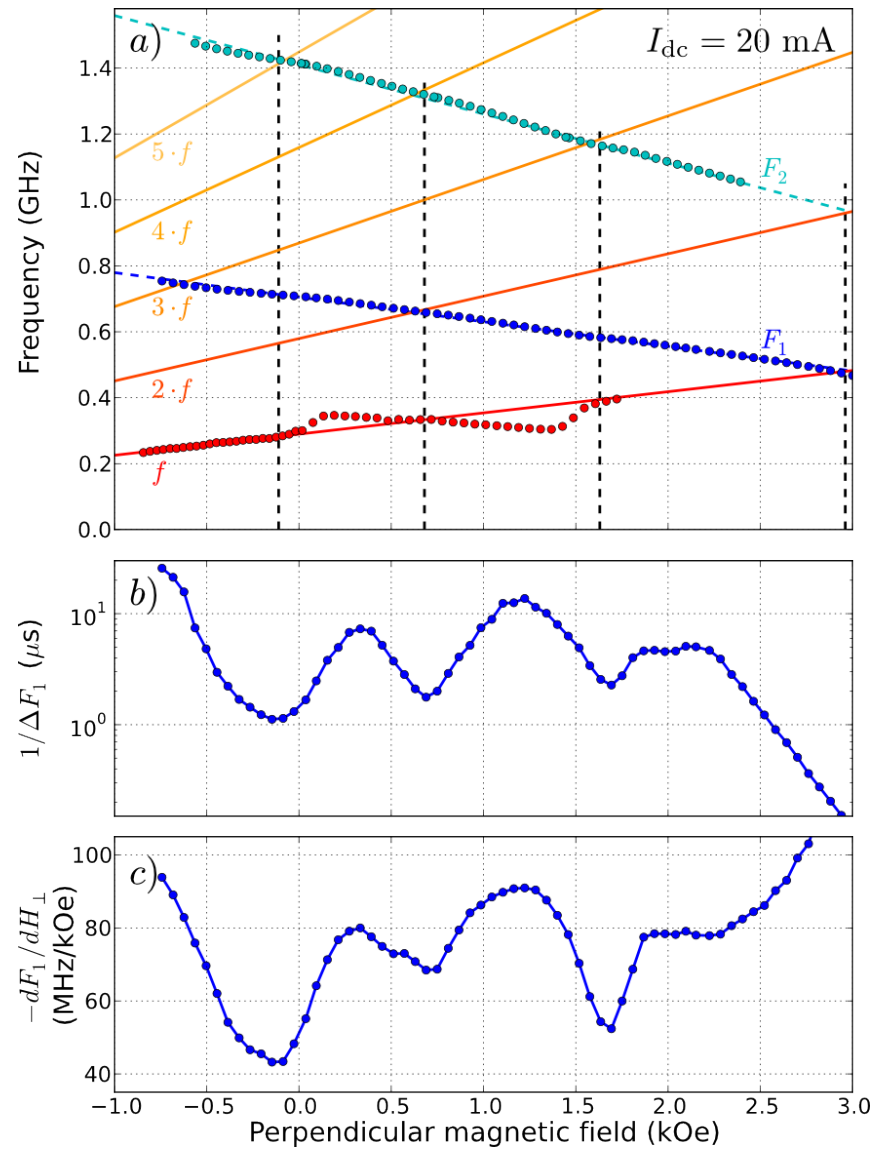

FIG. 3. (Color online) (a) Blue dots: fundamental frequency $F_{1}$ and harmonics $F_{2}$ of the auto-oscillating mode dominated by the thick layer (vortex core polarity $p_{\mathrm{B}}=-1$ ) as a function of $H_{\perp}$. Red dots: frequency $f$ of the overdamped mode dominated by the thin layer (polarity $p_{\mathrm{A}}=+1$ ). Red tone straight lines are guides to the eye and show successive harmonics of $f$. (b) Evolution of the inverse generation linewidth. (c) Slope of the oscillation frequency $F_{1}$ vs. $H_{\perp}$.

quasi-isochronicity: micromagnetic simulations have indeed shown that such terms can substantially reduce the nonlinear phase-amplitude coupling [26].

To elucidate the linewidth broadenings observed in Fig.2a, it should be noticed that in addition to the gyrotropic mode dominated by the thick $\mathrm{Py}_{\mathrm{B}}$ layer excited by STT, there is a gyrotropic mode dominated by the thin $\mathrm{Py}_{\mathrm{A}}$ layer, which for $I_{\mathrm{dc}}>0$ is overdamped by STT [25]. In order to probe this mode, we use a microwave antenna deposited on top of the sample which produces a microwave field $h_{\mathrm{rf}}$ in the plane of the Py layers [6, 20], and detect the dc voltage generated through the nanopillar when the vortex gyrotropic dynamics is excited at resonance by $h_{\mathrm{rf}}=3.6$ Oe [27]. We have reported the measured resonant frequency associated to the thin layer vortex mode as a function of $H_{\perp}$ in Fig, 3 a using red dots. The detailed analysis of the observed behavior is out of the scope of this Letter and will be reported separately. 
For the following demonstration, it is sufficient to use a linear approximation of it (red straight line). As expected, the gyrotropic mode frequency $f$ dominated by $\mathrm{Py}_{\mathrm{A}}$ is lower and has an opposite slope vs. $H_{\perp}$ than the one $F_{1}$ dominated by $\mathrm{Py}_{\mathrm{B}}$, due to smaller thickness $\left(L_{\mathrm{A}}<L_{\mathrm{B}}\right)$ and opposite core polarity $\left(p_{\mathrm{A}}=+1=-p_{\mathrm{B}}\right)$ [13. We also note that the linewidth of this mode ranges between 80 and $100 \mathrm{MHz}$, which is about 8 times larger than its natural linewidth, in agreement with the increase of relaxation of the thin layer due to STT.

Using red tone lines in Fig 3 a, we have plotted its harmonics $n=2$ to 5 together with the measured fundamental frequency $F_{1}$ and harmonics $F_{2}$ of the auto-oscillation signal (blue dots). We have also reported in Figs 3 b and 3 c the inverse generation linewidth (logarithmic scale) and the slope $-d F_{1} / d H_{\perp}$ of the oscillation frequency vs. $H_{\perp}$, respectively. At magnetic fields where the frequencies of the auto-oscillating and overdamped modes are commensurable, $p F_{1}=q f$, with $p, q \in \mathbb{N}$ (dashed vertical lines in Fig 3 a), a decrease of $-d F_{1} / d H_{\perp}$ concomitant with a decrease of the inverse linewidth is observed. We attribute this behavior to the interaction between the eigenmodes of the STNO. When they cross each other, their frequency dispersions soften [28] and some energy can be transferred from the auto-oscillating mode to the overdamped mode. As a result, this additional channel of relaxation leads to a decrease of the coherence time of the auto-oscillation (this effect is not taken into account in Eq.(1), which was derived in a single mode approximation [5]). Here, the dynamic dipolar interaction [29, 30] is an obvious source of coupling between modes, but we stress that the dissipative STT terms at play in the double vortex configuration can also be important 25. Finally, since the coupling strength depends on the difference in energy between the modes, we emphasize that the STNO should better be operated under conditions where large frequency gaps exist between the auto-oscillating mode and other modes in the system.

In conclusion, we have presented a STNO based on coupled vortices having its spectral purity and tuning sensitivity uncorrelated, which is unusual for STNOs. We have demonstrated that it is quasi-isochronous in a broad range of bias conditions, which suppresses the most stringent cause of broadening and leads to high spectral purity. The latter reaches its intrinsic value $Q>10000$ only when the overdamped mode do not interact with the auto-oscillating mode. We have also pointed out that $\nu \simeq 0$ can be due to the compensation of the magnetostatic and Oersted field contributions to the nonlinear frequency shift in our device. Interestingly for applications, this does not prevent large tunability thanks to the linear contribution of the current induced Oersted field to the confinement potential of the vortex cores.

We are greatly indebted to A. N. Slavin for fruitful discussions and for his support. This research was partly funded by the French ANR (grant SPINNOVA ANR-
11-NANO-0016) and the EU (FP7 grant MOSAIC ICTFP7-317950).

gregoire.deloubens@cea.fr

[1] J. Slonczewski, J. Magn. Magn. Mater. 159, L1 (1996).

[2] L. Berger, Phys. Rev. B 54, 9353 (1996).

[3] S. I. Kiselev, J. C. Sankey, I. N. Krivorotov, N. C. Emley, R. J. Schoelkopf, R. A. Buhrman, and D. C. Ralph, Nature 425, 380 (2003).

[4] W. H. Rippard, M. R. Pufall, S. Kaka, S. E. Russek, and T. J. Silva, Phys. Rev. Lett. 92, 027201 (2004).

[5] A. Slavin and V. Tiberkevich, IEEE Trans. Magn. 45, $1875(2009)$.

[6] A. Hamadeh, G. de Loubens, V. V. Naletov, J. Grollier, C. Ulysse, V. Cros, and O. Klein, Phys. Rev. B 85, 140408 (2012)

[7] J.-V. Kim, V. Tiberkevich, and A. N. Slavin, Phys. Rev. Lett. 100, 017207 (2008)

[8] V. S. Pribiag, I. N. Krivorotov, G. D. Fuchs, P. M. Braganca, O. Ozatay, J. C. Sankey, D. C. Ralph, and R. A. Buhrman, Nature Phys. 3, 498 (2007).

[9] A. Dussaux, B. Georges, J. Grollier, V. Cros, A. Khvalkovskiy, A. Fukushima, M. Konoto, H. Kubota, K. Yakushiji, S. Yuasa, K. Zvezdin, K. Ando, and A. Fert, Nat. Commun. 1, 8 (2010).

[10] M. Manfrini, T. Devolder, J.-V. Kim, P. Crozat, N. Zerounian, C. Chappert, W. V. Roy, L. Lagae, G. Hrkac, and T. Schrefl, Appl.Phys.Lett. 95, 192507 (2009).

[11] N. Locatelli, V. V. Naletov, J. Grollier, G. de Loubens, V. Cros, C. Deranlot, C. Ulysse, G. Faini, O. Klein, and A. Fert, Appl. Phys. Lett. 98, 062501 (2011).

[12] V. Sluka, A. Kákay, A. M. Deac, D. E. Bürgler, R. Hertel, and C. M. Schneider, Phys. Rev. B 86, 214422 (2012).

[13] G. de Loubens, A. Riegler, B. Pigeau, F. Lochner, F. Boust, K. Y. Guslienko, H. Hurdequint, L. W. Molenkamp, G. Schmidt, A. N. Slavin, V. S. Tiberkevich, N. Vukadinovic, and O. Klein, Phys. Rev. Lett. 102, 177602 (2009).

[14] A. V. Khvalkovskiy, J. Grollier, A. Dussaux, K. A. Zvezdin, and V. Cros, Phys. Rev. B 80, 140401 (2009).

[15] A. Dussaux, A. V. Khvalkovskiy, P. Bortolotti, J. Grollier, V. Cros, and A. Fert, Phys. Rev. B 86, 014402 (2012)

[16] K. Y. Guslienko, K. S. Buchanan, S. D. Bader, and V. Novosad, Appl. Phys. Lett. 86, 223112 (2005).

[17] N. Locatelli, A. E. Ekomasov, A. V. Khvalkovskiy, S. A. Azamatov, K. A. Zvezdin, J. Grollier, E. G. Ekomasov, and V. Cros, Appl. Phys. Lett. 102, 062401 (2013)

[18] M. Quinsat, V. Tiberkevich, D. Gusakova, A. Slavin, J. F. Sierra, U. Ebels, L. D. Buda-Prejbeanu, B. Dieny, M.-C. Cyrille, A. Zelster, and J. A. Katine, Phys. Rev. B 86, 104418 (2012)

[19] K. Y. Guslienko, Appl. Phys. Lett. 89, 022510 (2006).

[20] V. V. Naletov, G. de Loubens, G. Albuquerque, S. Borlenghi, V. Cros, G. Faini, J. Grollier, H. Hurdequint, N. Locatelli, B. Pigeau, A. N. Slavin, V. S. Tiberkevich, C. Ulysse, T. Valet, and O. Klein, Phys. Rev. B 84, 224423 (2011)

[21] A. V. Khvalkovskiy, J. Grollier, N. Locatelli, Y. V. Gorbunov, K. A. Zvezdin, and V. Cros, Appl. Phys. Lett. 
96, 212507 (2010).

[22] K. L. Metlov, arXiv:1308.0240.

[23] O. Sukhostavets, B. Pigeau, G. de Loubens, V. Naletov, O. Klein, K. Mitsuzuka, S. Andrieu, F. Montaigne, and K. Guslienko, arXiv:1307.5429.

[24] P. M. Braganca, O. J. Lee, O. Ozatay, L. Liu, G. Finocchio, D. C. Ralph, and R. A. Buhrman, Appl. Phys. Lett. 102, 252402 (2013).

[25] N. Locatelli, Dynamique par transfert de spin et synchronisation d'oscillateurs couplés à base de vortex magnétiques, Ph.D. thesis, Université Paris Sud (2012).

[26] D. Gusakova, M. Quinsat, J. F. Sierra, U. Ebels, B. Dieny, L. D. Buda-Prejbeanu, M.-C. Cyrille, V. Tiberke- vich, and A. N. Slavin, Appl. Phys. Lett. 99, 052501 (2011)

[27] S. Kasai, Y. Nakatani, K. Kobayashi, H. Kohno, and T. Ono, Phys. Rev. Lett. 97, 107204 (2006)

[28] B. Pigeau, C. Hahn, G. de Loubens, V. V. Naletov, O. Klein, K. Mitsuzuka, D. Lacour, M. Hehn, S. Andrieu, and F. Montaigne, Phys. Rev. Lett. 109, 247602 (2012)

[29] S. Sugimoto, Y. Fukuma, S. Kasai, T. Kimura, A. Barman, and Y. Otani, Phys. Rev. Lett. 106, 197203 (2011)

[30] P. S. Keatley, P. Gangmei, M. Dvornik, R. J. Hicken, J. Grollier, and C. Ulysse, Phys. Rev. Lett. 110, 187202 (2013). 